Physica Scripta, Vol. 77, № 6, art. 065004 (2008)

\title{
Accurate approximate solution to nonlinear oscillators in which the restoring force is inversely proportional to the dependent variable
}

A. Beléndez ${ }^{1}$, E. Gimeno ${ }^{1}$, E. Fernández ${ }^{2}$, D. I. Méndez ${ }^{1}$ and M.L. Alvarez ${ }^{1}$

${ }^{1}$ Departamento de Física, Ingeniería de Sistemas y Teoría de la Señal. Universidad de Alicante. Apartado 99. E-03080 Alicante. SPAIN

${ }^{2}$ Departamento de Óptica, Farmacología y Anatomía.

Universidad de Alicante. Apartado 99. E-03080 Alicante. SPAIN

Corresponding author: A. Beléndez

Phone: +34-96-5903651

Fax: +34-96-5903464 


\begin{abstract}
A modified generalized, rational harmonic balance method is used to construct approximate frequency-amplitude relations for a conservative nonlinear singular oscillator in which the restoring force is inversely proportional to the dependent variable. The procedure is used to solve the nonlinear differential equation approximately. The approximate frequency obtained using this procedure is more accurate than those obtained using other approximate methods and the discrepancy between the approximate frequency and the exact one is lower than $0.40 \%$.
\end{abstract}

Keywords: Nonlinear oscillators; Rational harmonic balance method; Analytical approximate solution.

PACS numbers: 02.30.Jr, 02.30.Lt, 02.30.-f 


\section{Introduction}

It is difficult to solve nonlinear differential equations and, in general, it is often more difficult to get an analytic approximation than a numerical one for a given nonlinear oscillatory system $[1,2]$. There is a large variety of approximate methods commonly used for solving nonlinear oscillatory systems including perturbations [1-8], variational [9], variational iteration [10-16], homotopy perturbation [17-32] or harmonic balance [2, 3338] methods, etc. Surveys of the literature with numerous references and useful bibliography and a review of these methods can be found in detail in [2] and [20]. In this paper we apply a modified generalized, rational harmonic balance method to obtain analytic approximate solutions for a nonlinear oscillator in which the restoring force is inversely proportional to the dependent variable This singular nonlinear oscillator has been recently studied by Mickens [39] and Beléndez et al. [40] using the standard harmonic balance method and by $\mathrm{He}$ [20] applying the homotopy perturbation method. This method can be applied to nonlinear oscillatory systems where the nonlinear terms are not small and no perturbation parameter is required. In this method the approximate solution obtained approximates all of the harmonics in the exact solution [38], whereas the usual harmonic balance techniques provide an approximation to only the lowest harmonic components. In an attempt to provide better solution methodology a modification in this technique is proposed. The approximate frequency derived here is more accurate and closer to the exact solution and the error in the resulting frequency is reduced and the maximum relative error is less than $0.4 \%$ for all values of $A$.

\section{Solution procedure}

Mickens [1] has recently analyzed the nonlinear differential equation

$$
\frac{d^{2} y}{d t^{2}}+\frac{1}{y}=0
$$

with initial conditions 


$$
y(0)=A, \quad\left(\frac{d y}{d t}\right)_{t=0}=0
$$

This equation occurs in the modelling of certain phenomena in plasma physics $[20,41]$. Mickens has also shown that all the motions corresponding to Eq. (1) are periodic [39]; the system will oscillate within symmetric bounds $[-A, A]$, and the angular frequency and corresponding periodic solution of the nonlinear oscillator are dependent on the amplitude $A$. Integration of Eq. (1) gives the first integral

$$
\frac{1}{2}\left(\frac{\mathrm{d} y}{\mathrm{~d} t}\right)^{2}+\log y=\log A
$$

where the integration constant was evaluated using the initial conditions of Eq. (2). From Eq. (3), the expression for the exact period, $T_{e x}(A)$, for the nonlinear oscillator given by Eq. (1) taking into account the initial conditions in Eq. (2) is

$$
T_{e x}(A)=4 \int_{0}^{A} \frac{d y}{\sqrt{2 \log (A / y)}}=4 \sqrt{2} A \int_{0}^{\infty} \mathrm{e}^{-t^{2}} d t=4 \sqrt{2} A \frac{\sqrt{\pi}}{2}=2 \sqrt{2 \pi} A
$$

The exact angular frequency is given by the expression

$$
\omega_{e x}(A)=\frac{2 \pi}{T_{e x}(A)}=\frac{2 \pi}{2 \sqrt{2 \pi} A}=\frac{\sqrt{2 \pi}}{2 A}=\frac{1.2533141}{A}
$$

In order to approximately solve Eq. (1), Mickens has rewritten this equation in a form that does not contain the $y^{-1}$ expression [39]

$$
y \frac{d^{2} y}{d t^{2}}+1=0
$$


and he solved this equation by applying the harmonic balance method. Following the lowest order harmonic balance method, a reasonable and simple initial approximation satisfying the conditions in Eq. (6) would be

$$
y_{1}(t)=A \cos \omega t
$$

and the solution for the angular frequency, $\omega_{M 1}(A)$ obtained by Mickens is

$$
\omega_{M 1}(A)=\frac{\sqrt{2}}{A}=\frac{1.414214}{A}
$$

and the percentage error of this approximate frequency in relation to the exact one is

$$
\text { percentage error }=\left|\frac{\omega_{e x}-\omega_{M 1}}{\omega_{e x}}\right| \cdot 100=12.8 \%
$$

Mickens [1] also used the second-order harmonic balance approximation

$$
y_{2}(t)=A_{2} \cos \omega t+B_{2} \cos 3 \omega t
$$

to the periodic solution of Eq. (6). Substitution of Eq. (10) into Eq. (6), simplifying the resulting expression and equating the coefficients of the lowest harmonics to zero gives two equations and taking into account that $A=A_{2}+B_{2}$, it is easy to obtain

$$
y_{M 2}(t)=\frac{10}{9} A \cos \left(\omega_{M 2} t\right)-\frac{1}{9} A \cos \left(3 \omega_{M 2} t\right)
$$

where the second-order approximate frequency, $\omega_{M 2}(A)$, is given by 


$$
\omega_{M 2}(A)=\frac{\sqrt{162}}{10 A}=\frac{1.272792}{A}
$$

and the percentage error is

$$
\text { percentage error }=\left|\frac{\omega_{e x}-\omega_{M 2}}{\omega_{e x}}\right| \cdot 100=1.6 \%
$$

The main objective of this paper is to solve Eq. (1) instead of Eq. (6) by applying a modified rational, generalized harmonic balance method. A new independent variable $\tau=\omega t$ is introduced. Then Eqs. (1) and (2) can be rewritten as

$$
\begin{aligned}
& \omega^{2} \frac{\mathrm{d}^{2} y(\tau)}{\mathrm{d} \tau^{2}}+\frac{1}{y(\tau)}=0 \\
& y(0)=A, \quad \frac{\mathrm{d} y}{\mathrm{~d} \tau}(0)=0
\end{aligned}
$$

The new independent variable is chosen in such a way that the solution of Eq. (14) is a periodic function of $\tau$ of period $2 \pi$. The corresponding frequency of the nonlinear oscillator is $\omega$ and it is a function of the initial amplitude $A$.

In order to determine an improved approximation we use a generalized, rational form given by the following expression $[1,38]$

$$
y_{2}(\tau)=\frac{A_{1} \cos \tau}{1+B_{2} \cos 2 \tau}
$$

In this equation $\omega$ is an approximation to the true angular frequency and $\left|B_{1}\right|<1$, and $A_{1}, B_{2}$ and $\omega$ are to be determined as functions of the initial conditions expressed in Eq. (4). 
From Eq. (15) we obtain $A_{1}=\left(1+B_{2}\right) A$ and Eq. (16) can be rewritten as follows

$$
y_{2}(\tau)=\frac{\left(1+B_{2}\right) A \cos \tau}{1+B_{2} \cos 2 \tau}
$$

The Fourier representation of Eq. (17) is

$$
y_{2}(\tau)=A \sum_{n=0}^{\infty} a_{2 n+1} \cos [(2 n+1) \tau]
$$

and the following result is obtained for the Fourier coefficients (see Appendix I)

$$
a_{2 n+1}=(-1)^{n} 2^{n+1}\left(\frac{B_{2}}{1-B_{2}}\right)^{n} \sqrt{\frac{1+B_{2}}{1-B_{2}}}\left(\frac{\sqrt{1-B_{2}}}{\sqrt{1-B_{2}}+\sqrt{1+B_{2}}}\right)^{2 n+1}
$$

Substituting Eq. (17) into Eq. (14) leads to

$$
\begin{aligned}
\frac{1+B_{2} \cos 2 \tau}{A\left(1+B_{2}\right) \cos \tau}+\omega^{2} \frac{4 A B_{2}\left(1+B_{2}\right) \cos \tau \cos 2 \tau}{\left(1+B_{2} \cos 2 \tau\right)^{2}}-\omega^{2} \frac{A\left(1+B_{2}\right) \cos \tau}{1+B_{2} \cos 2 \tau} \\
-\omega^{2} \frac{4 A B_{2}\left(1+B_{2}\right) \sin \tau \sin 2 \tau}{\left(1+B_{2} \cos 2 \tau\right)^{2}}+\omega^{2} \frac{8 A B_{2}^{2}\left(1+B_{2}\right) \cos \tau \sin ^{2} 2 \tau}{\left(1+B_{2} \cos 2 \tau\right)^{3}}=0
\end{aligned}
$$

Eq. (20) can be written as follows

$$
F\left(A, B_{2}, \omega, \tau\right)=0
$$

As $\left|B_{1}\right|<1$ we can do the following series expansion 


$$
F\left(A, B_{2}, \omega, \tau\right)=\sum_{n=0}^{\infty} F_{n}\left(A, B_{2}, \omega, \tau\right)=\sum_{n=0}^{\infty} f_{n}(A, \omega, \tau) B_{2}^{n}
$$

where

$$
f_{n}(A, \omega, \tau)=\frac{1}{n !}\left(\frac{\partial^{n} F\left(A, B_{2}, \omega, \tau\right)}{\partial B_{2}^{n}}\right)_{B_{2}=0}
$$

From Eq. (20) we can conclude that $f_{n}(A,-\tau)=-f_{n}(A, \tau)$.

Before applying the harmonic balance we truncate the series in Eq. (22) as follows

$$
F\left(A, B_{2}, \omega, \tau\right) \approx F^{(N)}\left(A, B_{2}, \omega, \tau\right)=\sum_{n=0}^{N} F_{n}\left(A, B_{2}, \omega, \tau\right)=\sum_{n=0}^{N} f_{n}(A, \omega, \tau) B_{2}^{n}
$$

and we approximately write Eq. (21) as

$$
F^{(N)}\left(A, B_{2}, \omega, \tau\right)=0
$$

We can consider different approaches taking into account different values of $N$. In the simplest case we have to consider $N=1$ and Eq. (24) yields

$$
\begin{aligned}
& F^{(1)}\left(A, B_{2}, \omega, \tau\right)=f_{0}(A, \omega, \tau)+f_{1}(A, \omega, \tau) B_{2}=-\omega^{2} A \cos \tau+\frac{1}{A \cos \tau} \\
& \quad+\left(-\omega^{2} A \cos \tau+5 \omega^{2} A \cos \tau \cos 2 \tau-\frac{1}{A \cos \tau}+\frac{\cos 2 \tau}{A \cos \tau}-4 \omega^{2} A \sin \tau \sin 2 \tau\right) B_{2}
\end{aligned}
$$

Expanding $F^{(1)}\left(A, B_{2}, \omega, \tau\right)$ in a trigonometric series, the following result is obtained

$$
F^{(1)}\left(A, B_{2}, \omega, \tau\right)=H_{1}^{(1)}\left(A, B_{2}, \omega\right) \cos \tau+H_{3}^{(1)}\left(A, B_{2}, \omega\right) \cos 3 \tau+H O H
$$

where 


$$
\begin{aligned}
H_{1}^{(1)}\left(A, B_{2}, \omega\right) & =\frac{4}{\pi} \int_{0}^{\pi / 2} F^{(1)}\left(A, B_{2}, \omega, \tau\right) \cos \tau d \tau \\
H_{3}^{(1)}\left(A, B_{2}, \omega\right) & =\frac{4}{\pi} \int_{0}^{\pi / 2} F^{(1)}\left(A, B_{2}, \omega, \tau\right) \cos 3 \tau d \tau
\end{aligned}
$$

Setting the coefficients of the two lowest harmonics, $\cos \tau$ and $\cos 3 \tau$, to zero in Eq. (27) we can obtain $B_{2}$ and the second order approximate frequency $\omega$ as a function of $A$. From Eqs. (26)-(29) we obtain

$$
\begin{gathered}
H_{1}^{(1)}\left(A, B_{2}, \omega\right)=\frac{2}{A}-\frac{2 B_{2}}{A}-A \omega^{2}=0 \\
H_{3}^{(1)}\left(A, B_{2}, \omega\right)=-\frac{2}{A}+\frac{4 B_{2}}{A}+\frac{9}{2} A B_{2} \omega^{2}=0
\end{gathered}
$$

Solving Eqs. (30) and (31) for $B_{2}$ and $\omega$ yields

$$
\begin{gathered}
B_{2}=\frac{1}{7}(6-\sqrt{22}) \approx 0.18083 \\
\omega_{2}^{(1)}(A)=\sqrt{\frac{4\left(1-B_{2}\right)}{2+B_{2}}} \frac{1}{A}=\sqrt{\frac{1+\sqrt{22}}{20-\sqrt{22}}} \frac{2}{A} \approx \frac{1.2193273}{A}
\end{gathered}
$$

The percentage error for this second order approximate frequency is

$$
\text { percentage error }=\left|\frac{\omega_{e x}-\omega_{2}^{(2)}}{\omega_{e x}}\right| \cdot 100=2.7 \%
$$

which is higher than the percentage error for the second approximate frequency obtained 
by Mickens (1.6\%) when the standard harmonic balance method is applied to Eq. (7).

The approximate frequency given in Eq. (33) is the same that the frequency obtained by applying the method proposed by $\mathrm{Wu}$ and Lim $[42,43]$ in which they combine the harmonic balance method and linearization of nonlinear oscillation equation (see Appendix II).

Therefore, the second approximation to the periodic solution of the nonlinear oscillator is given by the following equation

$$
\frac{y_{2}^{(1)}(t)}{A}=\frac{1.187083463 \cos \left(1.2193273 A^{-1} t\right)}{1+0.187083463 \cos \left(2.4386546 A^{-1} t\right)}
$$

This periodic solution has the following Fourier series expansion

$$
\begin{aligned}
\frac{y_{2}^{(1)}(t)}{A} & =\sum_{n=0}^{\infty} a_{2 n+1}^{(2)} \cos \left[(2 n+1) \omega_{2}^{(1)} t\right] \\
& =1.09437 \cos \omega_{2}^{(1)} t-0.103281 \cos 3 \omega_{2}^{(1)} t+0.0074718 \cos 5 \omega_{2}^{(1)} t-0.000919889 \cos 7 \omega_{2}^{(1)} t+\ldots
\end{aligned}
$$

where

$$
a_{2 n+1}^{(1)}=\frac{4}{\pi} \int_{0}^{\pi / 2} \frac{1.187083463 \cos \tau}{1+0.187083463 \cos 2 \tau} \cos [(2 n+1) \tau] d \tau
$$

As we can see, Eq. (35) gives an expression that approximates all of the harmonics in the exact solution whereas the usual harmonic balancing techniques provide and approximation to only the lowest harmonic components.

We can improve our results if we consider $N=2$ in Eq. (24) 


$$
\begin{aligned}
& F^{(2)}\left(A, B_{2}, \omega, \tau\right)=f_{0}(A, \omega, \tau)+f_{1}(A, \omega, \tau) B_{2}+f_{2}(A, \omega, \tau) B_{2}^{2}= \\
& \quad=-\omega^{2} A \cos \tau+\frac{1}{A \cos \tau}+\left(-\omega^{2} A \cos \tau+5 \omega^{2} A \cos \tau \cos 2 \tau-\frac{1}{A \cos \tau}+\frac{\cos 2 \tau}{A \cos \tau}\right. \\
& \left.\quad-4 \omega^{2} A \sin \tau \sin 2 \tau\right) B_{2}+\left(5 \omega^{2} A \cos \tau \cos 2 \tau-9 \omega^{2} A \cos \tau \cos ^{2} 2 \tau+\frac{1}{A \cos \tau}-\frac{\cos 2 \tau}{A \cos \tau}\right. \\
& \left.\quad-4 \omega^{2} A \sin \tau \sin 2 \tau+8 \omega^{2} A \cos 2 \tau \sin \tau \sin 2 \tau+8 \omega^{2} A \cos \tau \sin ^{2} 2 \tau\right) B_{2}^{2}
\end{aligned}
$$

Expanding $F^{(2)}\left(A, B_{2}, \omega, \tau\right)$ in a trigonometric series yields

$$
F^{(2)}\left(A, B_{2}, \omega, \tau\right)=H_{1}^{(2)}\left(A, B_{2}, \omega\right) \cos \tau+H_{3}^{(2)}\left(A, B_{2}, \omega\right) \cos 3 \tau+H O H
$$

where

$$
\begin{aligned}
& H_{1}^{(2)}\left(A, B_{2}, \omega\right)=\frac{4}{\pi} \int_{0}^{\pi / 2} F^{(2)}\left(A, B_{2}, \omega, \tau\right) \cos \tau d \tau \\
& H_{3}^{(2)}\left(A, B_{2}, \omega\right)=\frac{4}{\pi} \int_{0}^{\pi / 2} F^{(2)}\left(A, B_{2}, \omega, \tau\right) \cos 3 \tau d \tau
\end{aligned}
$$

Setting the coefficients of $\cos \tau$ and $\cos 3 \tau$ to zero in Eqs. (40)-(41) we can obtain $B_{2}$ and the second order aproximate frequency $\omega$ as a funtion of $A$. From Eqs. (38)-(41) we obtain

$$
\begin{gathered}
H_{1}^{(2)}\left(A, B_{2}, \omega\right)=\frac{2}{A}-\frac{2 B_{2}}{A}+\frac{2 B_{2}^{2}}{A}-A \omega^{2}-\frac{1}{2} A B_{2} \omega^{2}=0 \\
H_{3}^{(2)}\left(A, B_{2}, \omega\right)=-\frac{2}{A}+\frac{4 B_{2}}{A}-\frac{4 B_{2}^{2}}{A}+\frac{9}{2} A B_{2} \omega^{2}+\frac{9}{4} A B_{2}^{2} \omega^{2}=0
\end{gathered}
$$


Solving Eqs. (42) and (43) for $B_{2}$ and $\omega$ yield

$$
\begin{gathered}
B_{2}=\frac{1}{27}\left\lfloor 13-26\left(\frac{98}{-703+27 \sqrt{12641}}\right)^{1 / 3}+\left(\frac{-4921+189 \sqrt{1353}}{2}\right)^{1 / 3}\right\rfloor \approx 0.183131 \\
\omega_{2}^{(2)}(A)=\sqrt{\frac{4\left(1-B_{2}+B_{2}^{2}\right)}{2+B_{2}}} \frac{1}{A} \approx \frac{1.2482546}{A}
\end{gathered}
$$

The percentage error for the second order approximate frequency is

$$
\text { percentage error }=\left|\frac{\omega_{e x}-\omega_{2}^{(2)}}{\omega_{e x}}\right| \cdot 100=0.40 \%
$$

which is clearly lower than the percentage error for the second approximate frequency obtained by Mickens (1.6\%) when the standard harmonic balance method is applied to Eq. (7). By applying the method proposed by $\mathrm{Wu}$ and Lim with $N=3$, the value $1.2352721 / \mathrm{A}$ (percentage error, 1.44\%) for the approximate frequency is obtained [40]. Using this method and $N \rightarrow \infty$, the value $1.237330 / A$ (percentage error, 1.28\%) is obtained [40].

Therefore, the second approximation to the periodic solution of the nonlinear oscillator is given by the following equation

$$
\frac{y_{2}^{(2)}(t)}{A}=\frac{1.183131284 \cos \left(1.2482546 A^{-1} t\right)}{1+0.183131284 \cos \left(2.4965092 A^{-1} t\right)}
$$

This periodic solution has the following Fourier series expansion 


$$
\begin{aligned}
& \frac{y_{2}^{(2)}(t)}{A}=\sum_{n=0}^{\infty} a_{2 n+1} \cos \left[(2 n+1) \omega_{2}^{(2)} t\right] \\
& \quad=1.09235 \cos \omega_{2}^{(2)} t-0.100874 \cos 3 \omega_{2}^{(2)} t+0.0093154 \cos 5 \omega_{2}^{(2)} t-0.000860244 \cos 7 \omega_{2}^{(2)} t+\ldots
\end{aligned}
$$

where

$$
a_{2 n+1}^{(2)}=\frac{4}{\pi} \int_{0}^{\pi / 2} \frac{1.183131284 \cos \tau}{1+0.183131284 \cos 2 \tau} \cos [(2 n+1) \tau] d \tau
$$

Once again, we can see that Eq. (49) gives an expression that approximates all of the harmonics in the exact solution.

The normalized periodic exact solution, $y_{e x} / A$, achieved numerically integrating Eq. (1) and the second-order approximate solution obtained by Mickens, $y_{M 2} / A$ (Eq. (11)) is plotted, whereas the normalized periodic exact solution, $y_{e x} / A$, and the proposed secondorder approximate solution, $y_{2}^{(2)} / A$ (Eq. (47)) is plotted in Figure 2. In Figure 3 we plotted the differences $\Delta=\left(y_{e x}-y_{M 2}\right) / A$ and $\Delta=\left(y_{e x}-y_{2}^{(2)}\right) / A$. In these Figures $1-3, h$ is defined as follows

$$
h=\frac{t}{T_{e x}}
$$

Figures 1-3 show that Eqs. (45) and (47) can provide high accurate approximations to the exact frequency and the exact periodic solution. These results are an indication of the accuracy of the proposed modified generalized harmonic balance method as applied to this particular problem and show that it provides an excellent approximation to the solution of Eq. (1).

\section{Conclusions}

A modified generalized, rational harmonic balance method has been applied to obtain analytical approximate solutions for nonlinear oscillators in which the restoring force is 
inversely proportional to the dependent variable. The major conclusion is that this scheme provides excellent approximations to the solution of these nonlinear systems with high accuracy. The analytical representations obtained using this technique give excellent approximations to the exact solutions for the whole range of values of oscillation amplitude. These approximate solutions are better than the approximate solutions obtained using other approximate methods presented in the literature. For the second order approximation, the relative error of the analytical approximate frequency obtained using the approach considered in this paper is $0.40 \%$. The general conclusion is that the modified generalized, rational harmonic balance method provides an easy and direct procedure for determining the second-order analytical approximate solution of Eq. (1). This procedure also gives a very accurate estimate for the frequency. Finally, in comparison to the standard harmonic balance method, the present method produces a better solution.

\section{Appendix I}

From Eqs. (17) and (18) we can write

$$
\frac{\left(1+B_{2}\right) \cos \tau}{1+B_{2} \cos 2 \tau}=\sum_{n=0}^{\infty} a_{2 n+1} \cos [(2 n+1) \tau]
$$

Now applying the Taylor series expansion, it follows that

$$
\frac{\left(1+B_{2}\right) \cos \tau}{1+B_{2} \cos 2 \tau}=\left(\frac{1+B_{2}}{1-B_{2}}\right) \frac{\cos \tau}{1+\frac{2 B_{2}}{1-B_{2}} \cos ^{2} \tau}=\left(\frac{1+B_{2}}{1-B_{2}}\right) \sum_{m=0}^{\infty}(-1)^{m} 2^{m}\left(\frac{B_{2}}{1-B_{2}}\right)^{m} \cos ^{2 m+1} \tau
$$

The formula that allows us to obtain the odd power of the cosine is

$$
\cos ^{2 m+1} \tau=\frac{1}{2^{2 m}} \sum_{j=0}^{m}\left(\begin{array}{c}
2 m+1 \\
m-j
\end{array}\right) \cos [(2 j+1) \tau]
$$


Substituting Eq. (A3) into Eq. (A2) gives

$$
\frac{\left(1+B_{2}\right) \cos \tau}{1+B_{2} \cos 2 \tau}=\sum_{m=0}^{\infty}(-1)^{m} 2^{-m} \frac{\left(1+B_{2}\right) B_{2}^{m}}{\left(1-B_{2}\right)^{m+1}} \sum_{j=0}^{m}\left(\begin{array}{c}
2 m+1 \\
m-j
\end{array}\right) \cos [(2 j+1) \tau]
$$

Comparing Eqs. (A1) an (A4), we can find

$$
a_{2 n+1}=\sum_{m=n}^{\infty}(-1)^{m} 2^{-m} \frac{\left(1+B_{2}\right) B_{2}^{m}}{\left(1-B_{2}\right)^{m+1}}\left(\begin{array}{c}
2 m+1 \\
m-n
\end{array}\right)=(-1)^{n} 2^{n+1}\left(\frac{B_{2}}{1-B_{2}}\right)^{n} \sqrt{\frac{1+B_{2}}{1-B_{2}}}\left(\frac{\sqrt{1-B_{2}}}{\sqrt{1-B_{2}}+\sqrt{1+B_{2}}}\right)^{2 n+1}
$$

This result has been obtained using Mathematica ${ }^{\circledR}$.

\section{Appendix II}

We express the periodic solution to Eq. (1) with the assigned conditions in Eq. (2) in the form of

$$
y_{2}(\tau)=y_{1}(\tau)+u(\tau)
$$

where $u(\tau)$ is the correction part which is a periodic function of $\tau$ of period $2 \pi / \omega$ and

$$
u(0)=0, \quad\left(\frac{d u}{d t}\right)_{t=0}=0
$$

Substituting Eq. (A6) into Eq. (1) gives 


$$
\frac{\mathrm{d}^{2} y_{1}}{\mathrm{~d} \tau^{2}}+\frac{\mathrm{d}^{2} u}{\mathrm{~d} \tau^{2}}+\frac{1}{y_{1}(\tau)+u(\tau)}=0
$$

$\mathrm{Wu}$ and Lim $[42,43]$ presented an approach by combining the harmonic balance method and linearization of nonlinear oscillation equation with respect to displacement increment only, $u(t)$. This harmonic balance approach will be used to approximately solve Eq. (A8). Linearizing the governing Eq. (A8) with respect to the correction $u(\tau)$ at $y_{1}(\tau)$ leads to

$$
\frac{\mathrm{d}^{2} y_{1}}{\mathrm{~d} \tau^{2}}+\frac{\mathrm{d}^{2} u}{\mathrm{~d} \tau^{2}}+\frac{1}{y_{1}(\tau)}-\frac{u(\tau)}{y_{1}^{2}(\tau)}=0
$$

To obtain the second approximation to the solution, $u(\tau)$ in Eq. (A6), which must satisfy the initial conditions in Eq. (A7), we take into account the following second-order harmonic balance approximation

$$
y_{2}(\tau)=B_{1} \cos \tau+B_{3} \cos 3 \tau=B_{1} \cos \tau-B_{1} \cos \tau+B_{3} \cos 3 \tau=A \cos \tau+B_{3}(\cos 3 \tau-\cos \tau)
$$

where we have taken into account that $A=A_{2}+B_{2}$. From Eqs. (A6), and (A10) we can see that $u(\tau)$ takes the form

$$
u(\tau)=B_{3}(\cos 3 \tau-\cos \tau)
$$

where $B_{3}$ is a constant to be determined.

Substituting Eqs. (A10) and (A11) into Eq. (A9), expanding the expression in a trigonometric series and setting the coefficients of the terms $\cos \tau$ and $\cos 3 \tau$ equal to zero, respectively, leads to

$$
-\left(A-B_{3}\right) \omega^{2}+\frac{2}{A^{2}}\left(A+2 B_{3}\right)=0
$$

and 


$$
\frac{-2 A-8 B_{3}-9 B_{3} A^{2} \omega^{2}}{A^{2}}=0
$$

From Eqs. (A12) and (A13) we can obtain $B_{3}$ and $\omega_{2}$ as follows

$$
\begin{aligned}
& B_{3}=\frac{A}{14}(\sqrt{22}-6)=-0.093542 A \\
& \omega_{2}(A)=\sqrt{\frac{2 A+8 B_{3}}{-9 B_{3} A^{2}}}=\frac{1.2193273}{A}
\end{aligned}
$$

With this value for $B_{3}$, Eq. (A10) can be written

$$
\begin{aligned}
\frac{y_{2}(t)}{A} & =\frac{20-\sqrt{22}}{14} A \cos \omega_{2} t+\frac{\sqrt{22}-6}{14} A \cos 3 \omega_{2} t \\
& =1.093542 A \cos \omega_{2} t-0.093542 A \cos 3 \omega_{2} t
\end{aligned}
$$

which has only two harmonics.

\section{Acknowledgements}

This work was supported by the "Ministerio de Educación y Ciencia", Spain, under project FIS2005-05881-C02-02, and by the "Generalitat Valenciana", Spain, under project ACOMP/2007/020. 


\section{References}

[1] Mickens R E 1996 Oscillations in Planar Dynamics Systems (World Scientific, Singapore)

[2] He J H 2006 Non-perturbative methods for strongly nonlinear problems (dissertation.de-Verlag im Internet $\mathrm{GmbH}$, Berlin)

[3] Nayfeh A H 1985 Problems in Perturbations (Wiley, New York)

[4] He J H 2001 Modified Lindstedt-Poincare methods for some non-linear oscillations. Part III: double series expansion Int. J. Nonlinear Sci. Numer. Simul. 2 317-20

[5] He J H 2002 Modified Lindstedt-Poincare methods for some non-linear oscillations. Part I: expansion of a constant Int. J. Nonlinear Mech. 37 309-14

[6] He J H 2002 Modified Lindstedt-Poincare methods for some non-linear oscillations. Part II: a new transformation Int. J. Nonlinear Mech. 37 315-20

[7] Amore P and Fernández F M 2005 Exact and approximate expressions for the period of anharmonic oscillators Eur. J. Phys. 26 589-601

[8] Amore P, Raya A and Fernández F M 2005 Alternative perturbation approaches in classical mechanics Eur. J. Phys. 26 1057-63

[9] He J H 2007 Variational approach for nonlinear oscillators Chaos Solitons Fractals 34 1430-9

[10] He J H and Wu X H 2006 Construction of solitary solution and compact on-like solution by variational iteration method Chaos Solitons Fractals 29 108-13

[11] Dehghan M and Tatari M 2007 The use of He's variational iteration method for solving multipoint boundary value problems Phys. Scr. 72 672-6

[12] Tatari M and Dehghan M 2006 The use of He's variational iteration method for solving a Fokker-Planck equation Phys. Scr. 74 310-6

[13] Tatari M and Dehghan M 2007 On the convergence of He's variational iteration method J. Comput. Appl. Math. 207 121-8

[14] Tatari M and Dehghan M 2007 He's variational iteration method for computing a control parameter in a semi-linear inverse parabolic equation Chaos Solitons Fractals 33 671-7

[15] Tatari M and Dehghan M 2007 Solution of problems in calculus of variations via He's variational iteration method Phys. Lett. A 362 401-6 
[16] Miansari Mo., Ganji D D, and Miansari Me. 2008 Application of He's variational iteration method to nonlinear heat transfer equations Phys. Lett. A 372 779-85

[17] Dehghan M and Shakeri F 2007 Solution of a partial differential equation subject to temperature overspecification by He's homotopy perturbation method Phys. Scr. 75 $778-87$

[18] Chowdhury M S H and Hashim I 2007 Application of homotopy-perturbation method to nonlinear population dynamics models Phys. Lett. A 368 251-8

[19] Özis T and Yildirim A 2007 A comparative study of He's homotopy perturbation method for determining frequency-amplitude relation of a nonlinear oscillator with discontinuities Int. J. Nonlinear Sci. Numer. Simul. 8 243-8

[20] He J H 2006 Some asymptotic methods for strongly nonlinear equations Int. J. Mod. Phys. B 20 1141-99

[21] He J H 2006 New interpretation of homotopy perturbation method Int. J. Mod. Phys. B $202561-8$

[22] Dehghan M and Shakeri F 2008 Solution of an integro-differential equation arising in oscillating magnetic fields using He's homotopy perturbation method Progress in Electromagnetics Research 78 361-76

[23] Cai X C, Wu W Y and Li M S 2006 Approximate period solution for a kind of nonlinear oscillator by He's perturbation method Int. J. Nonlinear Sci. Numer. Simul. 7 109-17

[24] Chowdhury M S H and Hashim I 2007 Solutions of time-dependent Emden-Fowler type equations by homotopy-perturbation method Phys. Lett. A 368 305-13

[25] Beléndez A, Hernández A, Beléndez T, Fernández E, Álvarez M L and Neipp C 2007 Application of He's homotopy perturbation method to the Duffing-harmonic oscillator Int. J. Nonlinear Sci. Numer. Simul. 8 79-88

[26] Beléndez A, Hernández A, Beléndez T, Neipp C and Márquez A 2007 Application of the homotopy perturbation method to the nonlinear pendulum Eur. J. Phys. 28 93104

[27] Ganji D D and Sadighi A 2006 Application of He's homotopy-perturbation method to nonlinear coupled systems of reaction-diffusion equations Int. J. Nonlinear Sci. Numer. Simul. 7 411-8 
[28] Shakeri F and Dehghan M 2007 Solution of the delay differential equations via homotopy perturbation method Math. Comp. Mod. 46 1434-41

[29] Beléndez A, Pascual C, Gallego S, Ortuño M, Neipp C 2007 Application of a modified He's homotopy perturbation method to obtain higher-order approximations of a $x^{1 / 3}$ force nonlinear oscillator Phys. Lett. A 26, 421-6

[30] Shakeri F and Dehghan M 2007 Inverse problem of diffusion by He's homotopy perturbation method Phys. Scr. 75 551-6

[31] Beléndez A, Pascual C, Méndez D, Álvarez M L, Neipp C 2007 Application of He’s Homotopy Perturbation Method to the relativistic (an)harmonic oscillator. II: A More Accurate Approximate Solution Int. J. Non-linear Sci. Numer. Simul. 8 (4) 493-504

[32] Beléndez A, Pascual C, Fernández E, Neipp C and Beléndez T 2008 Higher-order approximate solutions to the relativistic and Duffing-harmonic oscillators by modified He's homotopy methods Phys. Scr. 77 (2), doi:10.1088/0031$8949 / 77 / 02 / 025004$

[33] Lim C W, Wu B S and Sun W P 2006 Higher accuracy analytical approximations to the Duffing-harmonic oscillator J. Sound Vib. 296 1039-45

[34] Beléndez A, Hernández A, Márquez A, Beléndez T and Neipp C 2006 Analytical approximations for the period of a simple pendulum Eur. J. Phys. 27 539-51

[35] Beléndez A, Pascual C 2007 Harmonic balance approach to the periodic solutions of the (an)harmonic relativistic oscillator Phys. Lett. A 371 291-9

[36] Beléndez A, Hernández A, Beléndez T, Álvarez M L, Gallego S, Ortuño M and Neipp C 2007 Application of the harmonic balance method to a nonlinear oscillator typified by a mass attached to a stretched wire J. Sound Vib. 302 1018-29

[37] Hu H and Tang J H 2006 Solution of a Duffing-harmonic oscillator by the method of harmonic balance J. Sound Vib. 294, 637-9

[38] Mickens R E and Semwogerere D 1996 Fourier analysis of a rational harmonic balance approximation for periodic solutions J. Sound Vib. 195 528-30

[39] Mickens R E 2007 Harmonic balance and iteration calculations of periodic solutions to $\ddot{y}+y^{-1}=0$ J. Sound Vib. 306, 968-72

[40] Beléndez A, Méndez D I, Beléndez T, Hernández A and Alvarez M Harmonic balance approaches to the nonlinear oscillators in which the restoring force is inversely proportional to the dependent variable J. Sound Vib. (2008) doi:10.1016/ 
j.jsv.2008.01.021

[41] Acton J R and Squire P T Solving Equations with Physical Understanding, Adam Hilger, Bristol and Boston, 1985 (Chapter 5)

[42] Wu B S and Lim C W 2004 Large amplitude nonlinear oscillations of a general conservative system Int. J. Non-linear Mech. 39 859-70

[43] Lim C W and Wu B S 2005 Accurate higher-order approximations to frequencies of nonlinear oscillators with fractional powers J. Sound Vib. 281 1157-62 


\section{FIGURE CAPTIONS}

Figure 1.- Comparison of normalized second order approximate solution obtained by Mickens using the standard harmonic balance method (circles and dashed line) with the exact solution (continuous line) as a function of $h$.

Figure 2.- Comparison of normalized second order approximate solution obtained using the modified generalized harmonic balance method presented in this paper (circles and dashed line) with the exact solution (continuous line) as a function of $h$.

Figure 3.- Difference between the normalized exact and second order approximate solution obtained using the standard harmonic balance method applied to Eq. (6) (black circles) and the modified generalized harmonic balance method presented in this paper applied to Eq. (1) (white circles), as a function of $h$. 


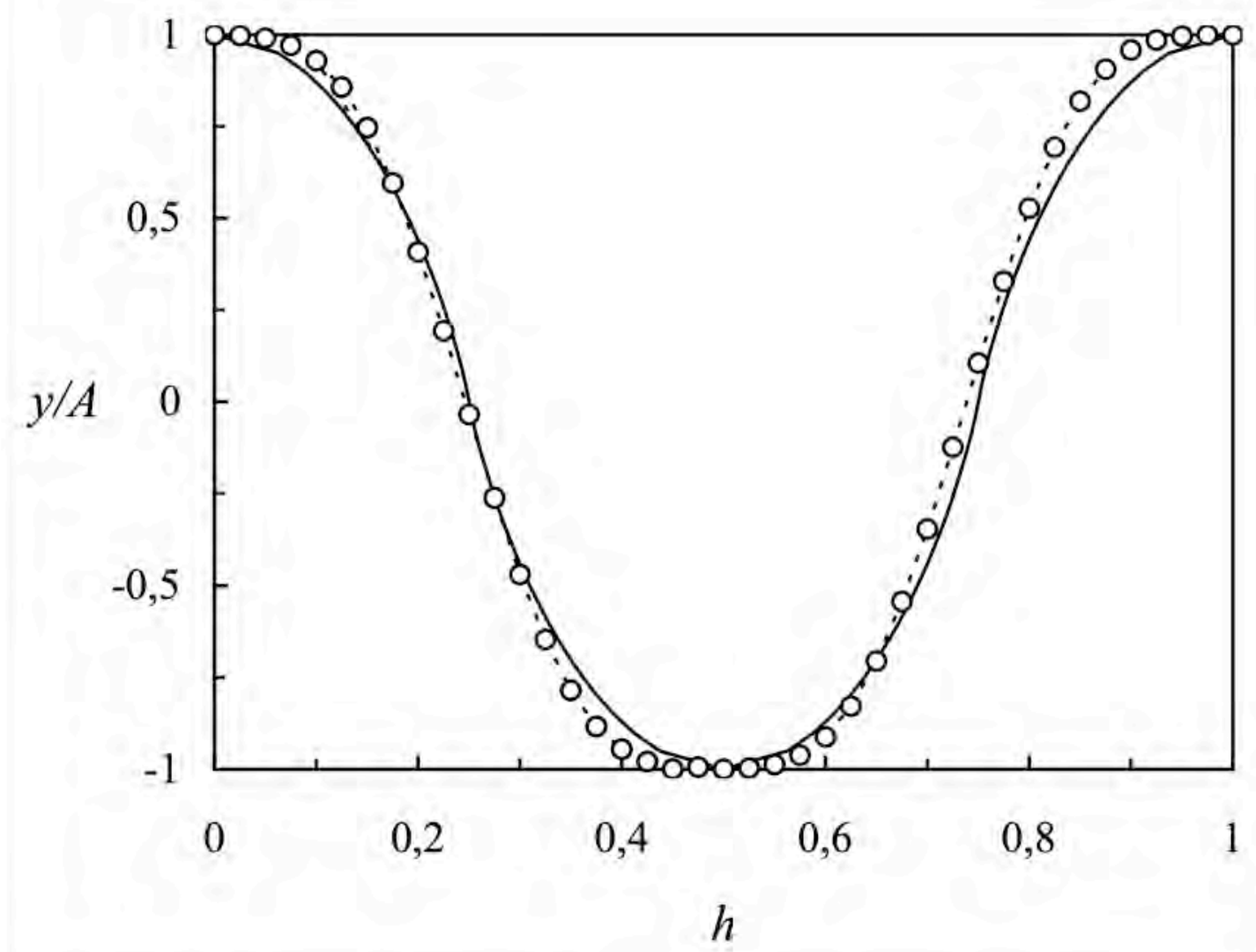

FIGURE 1 


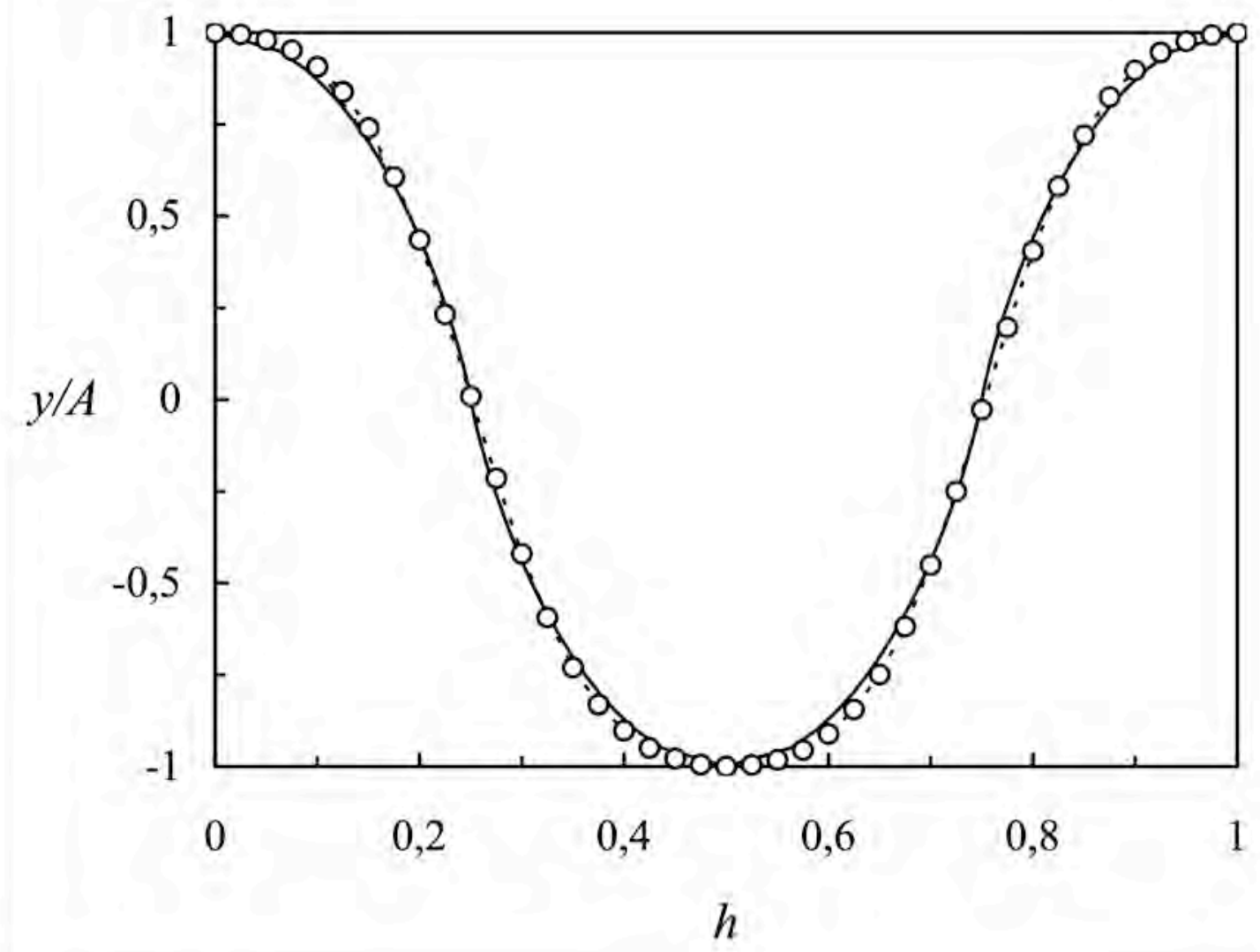

FIGURE 2 


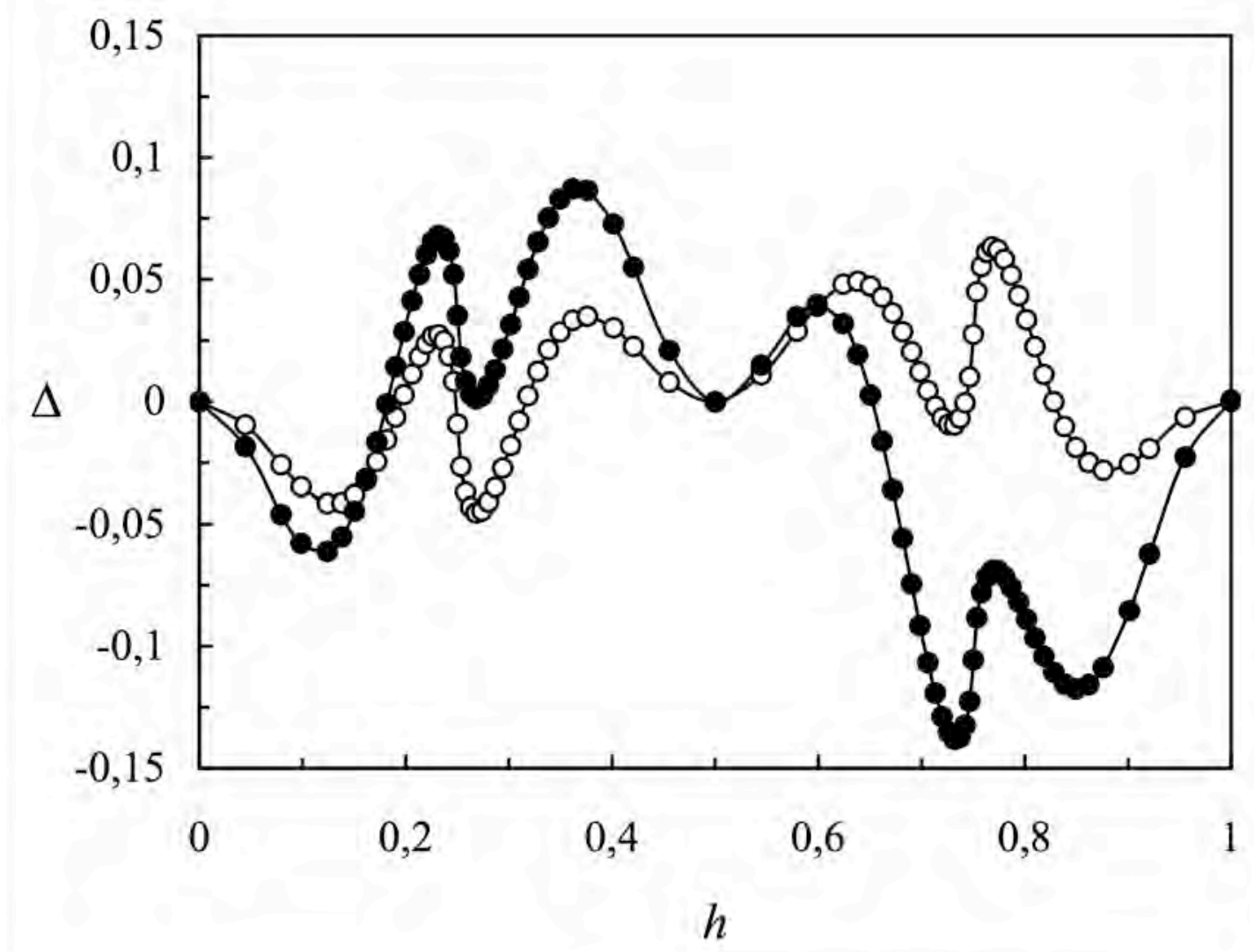

FIGURE 3 